\title{
A Neuropsychiatric Presentation of Seronegative Autoimmune Encephalitis: A Case Report
}

\author{
Muhammad Fahmi Ismaila, d, Eimer Moloneyb, Daniel J. Costello ${ }^{\mathrm{b}}$, \\ Brian J. Sweeney ${ }^{\mathrm{b}}$, Eugene M. Cassidy ${ }^{\mathrm{c}}$
}

\begin{abstract}
Autoimmune encephalitis with negative paraneoplastic, infectious and serology markers poses a considerable diagnostic and therapeutic challenge. We described a case with neuropsychiatric presentation of seronegative autoimmune encephalitis. Paraneoplastic, infectious and metabolic etiologies were excluded. The patient responded to immunotherapy and achieved full remission.
\end{abstract}

Keywords: Autoimmune encephalitis; Seronegative

\section{Introduction}

Autoimmune encephalitis is an acute inflammation of the brain secondary to autoimmune process that manifests as seizures, cognitive impairment, and neuropsychiatric symptoms [1]. Seronegative autoimmune encephalitis is a subgroup of encephalitis with suspected immunologic origin but with no identifiable pathogenic autoantibody [1].

\section{Case Report}

A 20-year-old female with no previous medical or psychiatric history was brought to the hospital following a cluster of epileptic seizures. Collateral history obtained suggested changes in personality and behavior from pre-morbid baseline which include disorganized behavior, increasing agitation and poor coping over a 4-week period. A brain CT was normal. An elec-

\footnotetext{
Manuscript accepted for publication September 17, 2015

a South Lee Mental Health Service, Cork University Hospital, Wilton, Cork, Ireland

bepartment of Neurology, Cork University Hospital, Wilton, Cork, Ireland 'Department of Psychiatry, School of Medicine, University College Cork, Cork, Ireland

${ }^{\mathrm{d}}$ Corresponding Author: Muhammad Fahmi Ismail, Acute Mental Health Unit, Cork University Hospital, Wilton, Cork, Ireland.

Email: dr.fahmi.ismail@gmail.com
}

doi: http://dx.doi.org/10.14740/jmc2297w troencephalography (EEG) showed moderately diffuse slowing. Cerebrospinal fluid (CSF) analysis revealed a lymphocytic pleocytosis with glucose and protein within the normal range. Her serum and CSF were sent to screen for infectious, inflammatory, autoimmune and metabolic cause of encephalitis which were all subsequently negative (Table 1 ). She was commenced on levetiracetam and intravenous acyclovir (Fig. 1).

On day 2, her symptoms deteriorated rapidly. She evolved into a state of mutism, unpredictable episodes of extreme agitation and a dramatic deterioration in social conduct. Further EEG studies and brain imaging by magnetic resonance imaging (MRI) were unremarkable. She was commenced on intravenous methylprednisolone on day 2.

On day 3 , her symptoms deteriorated further. She became increasingly confused with intermittent episodes of psychomotor excitation and abrupt stereotyped movement. She was assessed by the liaison psychiatry team. On mental state examination, the patient was sitting abnormally still with minimal interaction. She was verbally unresponsive to majority of the questions. She had a fixed stare with occasional shifts of attention. There was no evidence of posturing. When she did verbalize, there was evidence of intermittent echolalia. There was evidence of abrupt repetitive motor activity, i.e. abruptly standing on the bed followed by tumbling forwards. There was evidence of automatic obedience and ambitendency, i.e. she was observed to be stuck in mid-way from standing to sitting position for several minutes. She scored 16 out 69 on a BushFrancis catatonia rating scale.

Her condition deteriorated further on day 12 with worsening agitation which required sedation to manage the challenging behavior due to risk of self-injury. She was commenced on regular olanzapine at this stage. Methyprednisolone was stopped after 5 days and was switched to oral prednisolone. On day 9, she was commenced on intravenous immunoglobulins for 5 days with no response. On day 15, she developed symptoms of autonomic instability which include episodes of bradycardia, tachycardia, and hypoventilation. She was then transferred to the intensive care unit (ICU) in which she required intubation and full assisted ventilation. In ICU, she had five sessions of plasmapheresis with a trial of weekly rituximab. She responded well to the combination of plasmapheresis and rituximab. There was a difficulty in weaning off sedation initially due to her challenging behavior which required highdose olanzapine. She gradually improved and was transferred 
Table 1. List of Investigations and Results

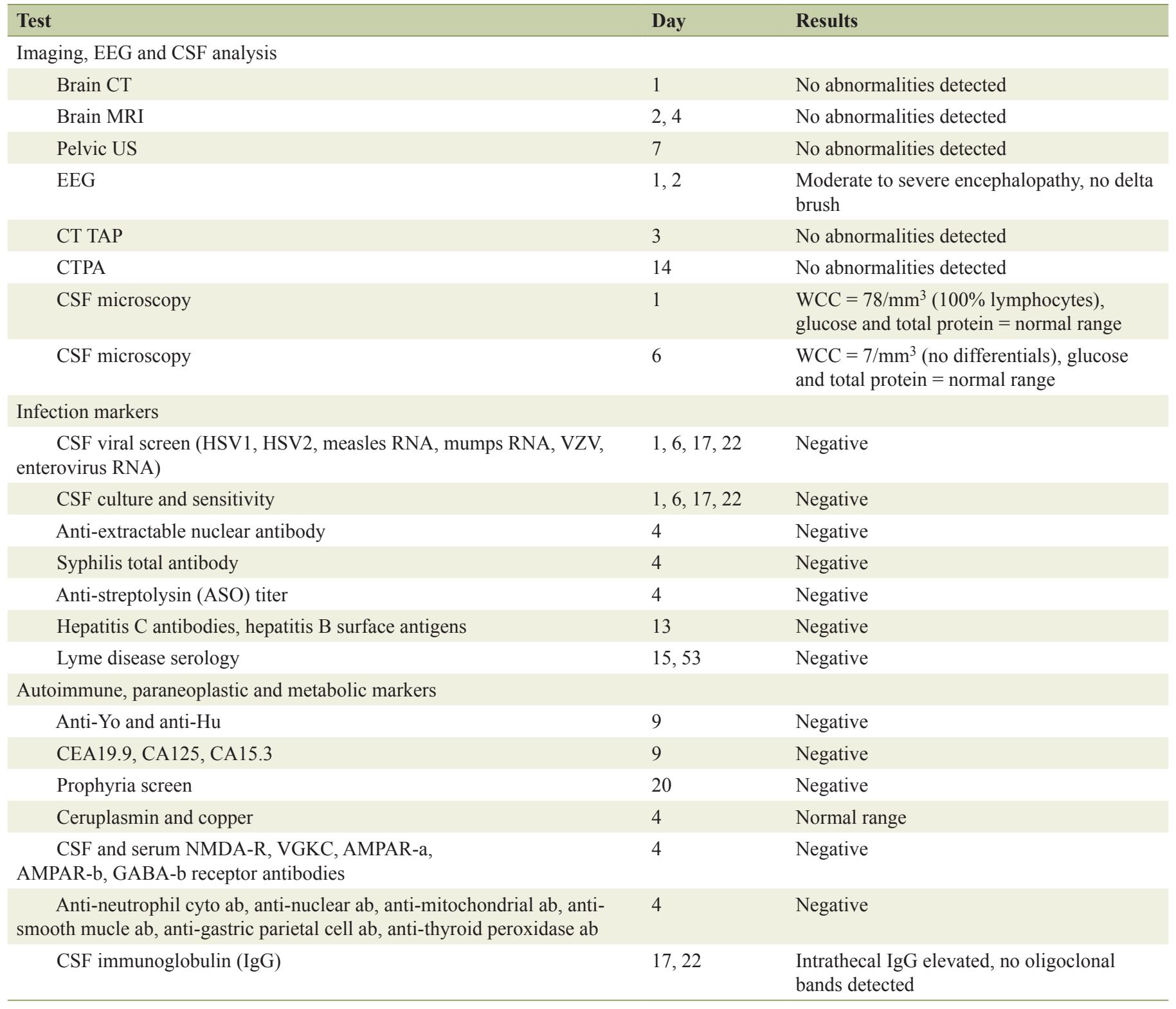

MRI: magnetic resonance imaging; EEG: electroencephalography; US: ultrasound; CT TAP: CT thorax, abdomen, and pelvis; CTPA: CT pulmonary angiography; CSF: cerebrospinal fluid; WCC: white cell count; HSV: herpes simplex virus; RNA: ribonucleic acid; VZV: varicella zoster virus.

to the ward after 32 days in ICU. On re-assessment, she had residual emotional lability with poor abstraction. She received full score on Addenbrooke's cognitive assessment (ACE-R). She was discharged from hospital on day 62 on prophylactic antiviral, reducing prednisolone, levetiracetam and olanzapine. She was reviewed 10 days post-discharge. Her mental state was stable, and had improved concentration with normal behavior. The olanzapine and prednisolone were gradually weaned.

\section{Discussion}

In $60 \%$ of cases of encephalitis, the cause remains unknown
[2]. Certain types of autoimmune encephalitis can be diagnosed by identifying a specific antibody in the serum or CSF (i.e. NMDAR, AMPAR, GABABR, LGI1, and CASPR2) [2]. New antibodies against neuronal cell surface or synaptic proteins have been continuously expanded for the last number of years [2].

Most autoimmune encephalitis is associated with CSF lymphocytic pleocytosis [3]. The mainstay of treatment for autoimmune encephalitis is immunotherapy which includes steroids, intravenous immunoglobulins, biologics and plasmapheresis [4]. Some have proposed a specific treatment pathway in those with suspected autoimmune encephalitis with negative auto-antibodies [5]. 


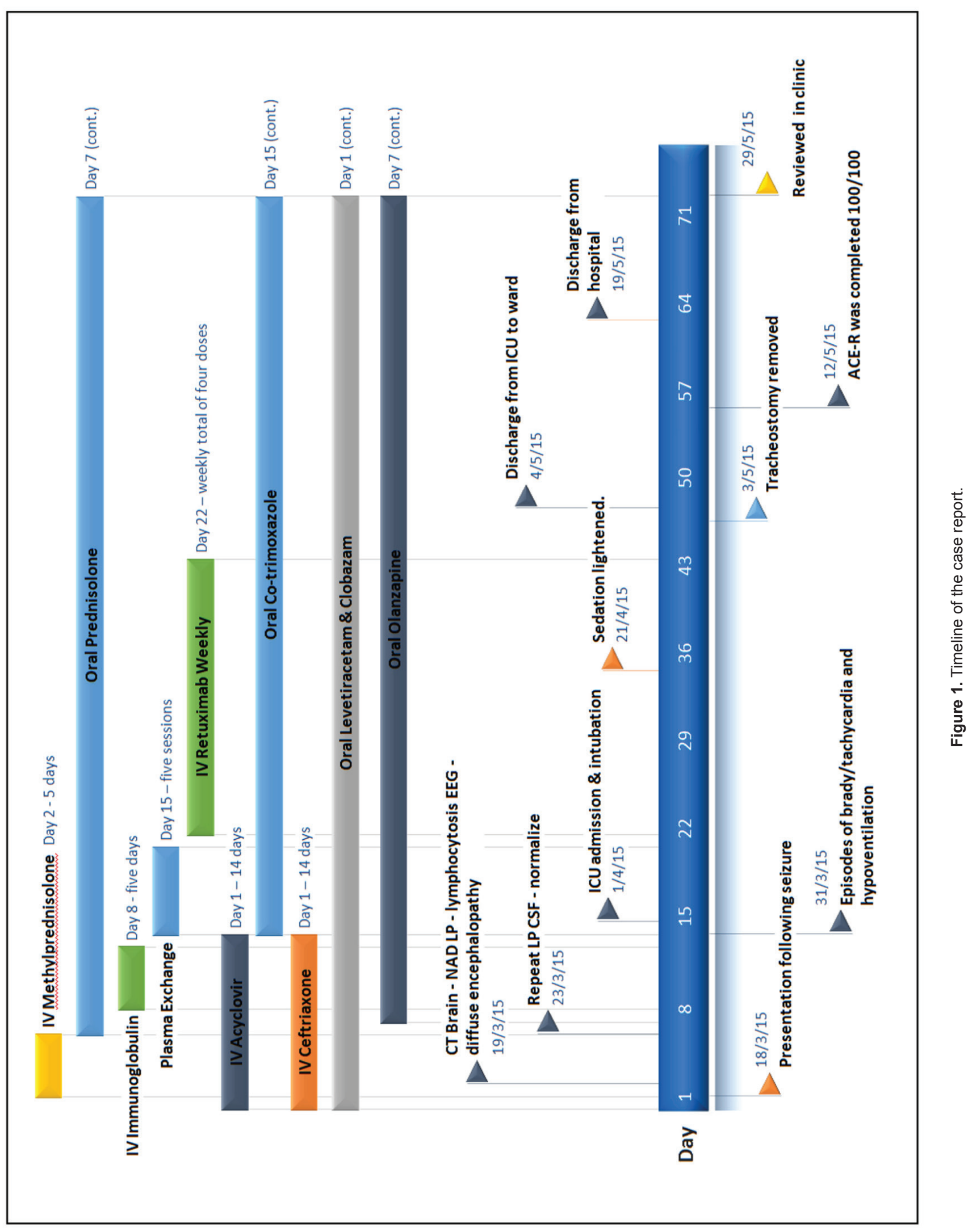




\section{Grant Support}

None.

\section{Conflict of Interest}

None.

\section{References}

1. Najjar S, Pearlman D, Devinsky O, Najjar A, Nadkarni S, Butler T, Zagzag D. Neuropsychiatric autoimmune encephalitis without VGKC-complex, NMDAR, and GAD autoantibodies: case report and literature review. Cogn
Behav Neurol. 2013;26(1):36-49.

2. Armangue T, Leypoldt F, Dalmau J. Autoimmune encephalitis as differential diagnosis of infectious encephalitis. Curr Opin Neurol. 2014;27(3):361-368.

3. Gable MS, Gavali S, Radner A, Tilley DH, Lee B, Dyner L, Collins A, et al. Anti-NMDA receptor encephalitis: report of ten cases and comparison with viral encephalitis. Eur J Clin Microbiol Infect Dis. 2009;28(12):14211429.

4. Mann AP, Grebenciucova E, Lukas RV. Anti-N-methylD-aspartate-receptor encephalitis: diagnosis, optimal management, and challenges. Ther Clin Risk Manag. 2014; 10:517-525.

5. Ahmad SA, Archer HA, Rice CM, Gerhand S, Bradley M, Wilkins A. Seronegative limbic encephalitis: case report, literature review and proposed treatment algorithm. Pract Neurol. 2011;11(6):355-361. 